\title{
A Concise Summary of Coagulation in Liver Disease
}

\author{
A Strachan*, L Abeysundara and SV Mallett \\ Department of Anaesthesia, Royal Free Hospital, United Kingdom
}

Submission: February 28, 2018; Published: March 20, 2018

*Corresponding author: A Strachan, Department of Anaesthesia, Royal Free Hospital, Royal Free Perioperative Research Group, London, Tel: 2037582000; Email: alexa.strachan@nhs.net

\begin{abstract}
Balanced haemostasis is dependent on the complex interaction of procoagulant and anticoagulant factors, fibrinolytic proteins, platelets and vascular endothelial component. The liver plays a key role in coagulation and as such chronic liver disease can have a profound impact on the interplay of these components. The net result is a fragile rebalancing in the haemostatic profile of liver disease patients as a result of decreased synthesis of both pro and anticoagulant factors. However, due to the reduced haemostatic reserve, this balance can be easily tipped into a thrombotic or bleeding state. Conventional coagulation tests such as PT/INR reflect only the decreased synthesis of pro-coagulant factors and do not adequately reflect the bleeding or thrombotic risk in these patients. As a consequence, an intrinsic hypercoagulable state is often underestimated in patients with liver disease. Point of care tests, specifically viscoelastic tests, can illustrate the haemostatic changes that are seen in liver disease and guide clinical management supporting the use, or indeed avoidance, of blood products.
\end{abstract}

Keywords: Liver disease; Cirrhosis; Haemostasis; Coagulation; Viscoelastic tests

Abbreviations: tPA: Tissue Plasminogen Activator; CLD: Chronic Liver Disease; vWF: Willebrand Factor; TFPI: Tissue Factor Pathway Inhibitor; CCTs: Conventional Coagulation Tests; ACT: Activated Clotting Time; VETs: Viscoelastic Tests; ROTEM: Rotational Thromboelastometry

\section{Introduction}

The cell-based model of coagulation depicts the complex interactions of pro and anticoagulant factors, fibrinolytic proteins, platelets and vascular endothelial components that are required for balanced haemostasis to take place. The liver plays a key role in haemostasis as hepatic parenchymal cells synthesise the majority of coagulation factors, anticoagulant proteins and constituents of the fibrinolytic system. Liver disease can consequently have a profound impact on the interplay of these components and impair haemostatic competence [1].

\section{Discussion}

\section{Haemostasis in Chronic Liver Disease}

The liver produces the vitamin $\mathrm{K}$ dependent factors II, VII, IX, $\mathrm{X}$, protein $\mathrm{C}$, protein $\mathrm{S}$ and protein $\mathrm{Z}$, in addition to factor $\mathrm{V}$, XIII, fibrinogen, antithrombin, $\alpha 2$-PI (plasminogen inhibitor) and plasminogen. The hepatic reticuloendothelial system aids regulation of coagulation by the clearance of activated clotting and fibrinolytic products, most notably tissue plasminogen activator (tPA) which is often elevated in liver disease [2]. Other coagulation factors produced outside the liver include von Willebrand factor (vWF), tPA, thrombomodulin, tissue factor pathway inhibitor (TFPI) and urokinase. Chronic liver disease (CLD) leads to various changes to the coagulation system. Impaired protein synthesis associated with liver disease results in reduced levels of the majority of hepatic coagulation factors, [3] the severity of which is coupled to disease progression. The exception is factor VIII and fibrinogen. Hepatic injury stimulates the production of factor VIII by sinusoidal endothelial cells and, in combination with reduced clearance of the circulating factor VIII/vWF-complex, results in normal or elevated vWF and factor VIII levels. Endogenous anticoagulant factors, protein $\mathrm{C}$, protein $\mathrm{S}$ and antithrombin, are reduced in cirrhosis to a similar extent as is seen with inherited deficiencies [1]. Fibrinogen promotes formation of stable clots and, as an acute phase reactant, fibrinogen levels may be preserved in CLD [4]. Moreover, evidence suggests that defective circulating fibrinogen does not necessarily translate into a bleeding risk [5]. In cirrhosis, changes in the makeup of fibrinogen lead to impaired fibrin polymerisation, resulting in structural changes to the fibrin clot. Although slower clot kinetics are observed in some patients with CLD, these clots have reduced permeability and consequently impaired lysis, suggesting that this procoagulant clot structure may predispose patients to a thrombotic state [5].

Impaired production and altered clearance of other factors within the fibrinolytic system also occur in CLD; most notably 
there is an imbalance between tPA and its principal inhibitor, plasminogen activator inhibitor (PAI-1), in addition to reduced levels of thrombin activatable fibrinolysis inhibitor (TAFI) which downregulates thrombin formation by inhibiting the complex of tissue factor (TF) and VIIa [1]. In clinical practice, fibrinolytic activity demonstrates significant variability between patients [6]. Platelets play a key role in the initiation of the coagulation cascade and in overall clot strength. Quantitative and qualitative reductions in platelet function are frequently seen in CLD [7]. The mechanisms responsible for this are numerous but include decreased thrombopoietin, splenic sequestration, immune-related platelet destruction and abnormalities in platelet aggregation [1]. Circulating endotoxin, commonly found in CLD, can impair platelet function and in addition, the endothelial release of natural anticoagulants (endogenous heparins), resulting from infection or physiological stress, can compound the ensuing coagulopathy [8]. In contrast, increased levels of vWF and its reduced cleavage by ADAMTS13 [4] results in platelet hyper responsiveness, evidenced by elevated markers of platelet activation. This may counterbalance other defective areas in the coagulation cascade. Platelet count is a rudimental assessment of the contribution of platelets to primary haemostasis and, although the contribution of platelet count to bleeding risk is stronger than that of the international normalised ratio (INR), a definitive cut-off point has yet to be conclusively defined. A platelet count $<60$ x109/L is commonly quoted as an indicator of increased risk of periprocedural bleeding [6]. A recent study of 318 patients with endstage liver failure undergoing teeth extractions, however, found platelet counts $>40 \times 109 / \mathrm{L}$ with an INR $<2.5$ to be associated with a low risk of bleeding episodes [9]. It is increasingly appreciated that in the presence of normal/high fibrinogen levels clot strength is preserved despite relatively low platelet counts [10].

The overall result of the changes that occur within the haemostatic system in advanced liver disease are described as "rebalanced" albeit with a markedly reduced functional reserve that is easily swayed to a state of thrombosis or excessive bleeding [4]. It is increasingly recognised that in CLD there is an underestimated intrinsic hypercoagulable state that is not elucidated by conventional coagulation tests (CCTs). Thrombotic events, for instance portal vein thrombosis, are common and although a prothrombotic state is not the only causative factor, it certainly contributes [3]. The overall thromboembolic rate reported in a recent UK audit was 3\% [4] and patients with CLD are at a higher risk of thrombosis than those without liver disease. This is supported by clinical observations that despite universal CCT abnormalities suggesting hypocoagulability, CLD patients tolerate invasive procedures without significant blood loss or need for transfusion [4]. Transfusion-free liver transplantation is achievable with at least $30 \%$ reported as not requiring intraoperative blood products [11] and clear pro-thrombotic post-operative tendencies have been demonstrated in patients undergoing major hepatic resections [12]. Furthermore, microvascular thrombi are associated with the progression of liver fibrosis in CLD [3]. A study investigating low-molecular-weight heparin on the formation of portal vein thrombosis in CLD showed a reduction in disease decompensation and mortality [5]. Different aetiologies of CLD have notable differences in their haemostatic profile which is not delineated by CCTs [4]. Cholestastic disorders are associated with hypercoagulability with a less pronounced effect on the anticoagulant pathways [14] and this, in conjunction with platelet hyperactivity, [15] can predispose to thrombosis. Non-alcoholic steatohepatitis patients have, amongst other changes, high PAI1 which can result in a systemic thrombotic predisposition [16]. Nonetheless, misconceptions about an 'auto-anticoagulated state', based on abnormalities in CCTs, persist among the medical profession. The resulting suboptimal thromboembolic prevention in patients with cirrhosis continues to place them at risk of fatal and non-fatal venous thromboembolic (VTE) events and must be addressed [13].

\section{Haemostasis in Acute Liver Disease}

Dysregulation of the anticoagulant and procoagulant pathways produces the complex coagulopathy seen in acute liver failure (ALF) [1]. The failing liver is unable to synthesise all coagulation factors but the acute phase reactants, factor VIII and vWF, are significantly increased by the inflammatory process. The principle coagulation activator, tissue factor, is increased promoting thrombin formation [4]. Studies examining viscoelastic tests in ALF have shown preserved or increased coagulation capability rising in parallel to disease severity [3]. In ALF, there is a state of functional hypofibrinogenemia with demonstrable reduction in the contribution to clot strength by fibrinogen on TEG functional assays as compared to normal. The fibrinolytic pathways are also altered with elevated expression of plasminogen activator inhibitor predisposing to thrombosis [4]. These findings are supported clinically where spontaneous or procedural bleeding is infrequent, regardless of a high INR, and microvascular thrombi occur. Endogenous heparin production is common in ALF due to endothelial injury. In such cases, the underlying global coagulation status can be analysed using heparinase-modified viscoelastic assays but CCTs are unable to describe this. INR does continue to have a role in disease prognostication in ALF [3].

\section{Conventional Coagulation Tests (CCTs)}

Traditional coagulation tests such as prothrombin time (PT) and INR measure the activity of procoagulant plasma proteins, characterising the initiation phase of coagulation [17], but do not reflect the reduction in the anticoagulant factors seen in liver disease. Additionally, CCTs are terminated at the start of fibrin polymerisation which occurs when less than $5 \%$ of thrombin has been generated [6]. Despite the use of phospholipid as a platelet membrane substitute, performing these tests on platelet poor plasma fails to capture the synergistic interaction of the entire coagulation cascade [4]. Furthermore; CCTs do not assess the activity of fibrinolytic pathways. Consequently, CCTs are unable to accurately describe the true in-vivo coagulation status of patients with liver disease and it is increasingly recognised that 
they are poor indicators of bleeding risk in this group of patients $[4,6]$. This is clinically reflected in studies demonstrating that preoperative INR is not a predictor for intra-operative transfusion in liver transplantation [18]. In addition, the turn-around time for CCTs which may be in excess of 60 minutes, limits their clinical usefulness in situations where surgery or bleeding leads to rapidly evolving haemostatic changes [4].

\section{Point-of-care testing and Viscoelastic testing}

Point of care tests (POCT) can facilitate real-time bedside measurement of haemoglobin concentration, platelet count, basic coagulation assessment (INR, PT, activated clotting time (ACT)) alongside blood gas analysis [4]. By providing rapidly measurable validated results, POCT have been particularly useful in anaesthesia and intensive care in assessing components of haemostasis and providing a perioperative guide for goal-directed clinical management. Assessment of global coagulation dynamics using viscoelastic tests (VETs) such as thromboelastography (TEG) and rotational thromboelastometry (ROTEM) uses whole blood to provide information on real-time clot dynamics from initial clot formation to final clot strength and dissolution [3]. VETs have been vital to our understanding of the complex interplay of haemostatic factors in liver disease and correlate well with bleeding risk. In both chronic and acute liver disease, VETs often display normal parameters supporting the haemostatic "rebalancing" that occurs and can reveal evidence of hypercoagulability [17]. Several studies suggest that the maximum amplitude (MA) of a TEG trace has a positive predictive value in liver transplantation for post-operative thromboembolic events [17]. Specific fibrinogen VET assays are available, which correlate well to the gold-standard Clauss fibrinogen in most circumstances [4] although caution should be exercised at low fibrinogen levels [17]. Clot strength is an amalgamation of fibrinogen and platelet activity and the contribution of each of these components can be assessed using VET parameters, permitting targeted therapeutic intervention. Platelet function VET assays are also available to evaluate the effect of antiplatelet agents [4]. Additionally, VETs can identify the presence of endogenous heparin-like substances released with endothelial damage with acute inflammation [3]. Recent work has outlined the potential role of assessing thrombin generation using velocity curves derived from TEG traces. Given the scarcity of thrombin generation assays in clinical practice, this shows promise but requires further prospective evaluation [19]. The utilisation of VET guided algorithms has reduced blood transfusion in multiple studies. A review of 17 studies with 1400 mostly surgical cardiac patients showed a reduction in transfusion and overall blood loss in massive haemorrhage. VET guided transfusion has been shown to result in a higher number of quality-of-life years gained in liver transplantation compared to standard practice and have been shown to be cost-effective [4]. The use of VET guided management of bleeding patients in liver transplantation is recommended in best practice guidelines [20] and there is increasing interest in utilising these tests to avoid unnecessary precautionary transfusions [21].

\section{Conclusion}

Liver disease is associated with complex alterations in clotting factors, resulting in a 're-balanced' haemostatic system. While overall functional coagulation capacity is preserved in stable liver disease, the balance is easily tipped during periods of physiological stress, resulting in an increased risk of both haemorrhagic and thrombotic complications. Assessment of coagulation status using conventional laboratory coagulation tests does not reflect the haemostatic alterations or bleeding risk. Viscoelastic tests of coagulation evaluate global haemostasis more accurately in this patient group.

\section{Conflict of Interest}

The authors declare no conflict of interest. No financial support was provided for this article.

\section{References}

1. De Sancho M, Pastores SM (2007) The Liver and Coagulation. Textbook of Hepatology 87(Suppl 1): 43-46.

2. Tripodi A, Mannucci PM (2011) The coagulopathy of chronic liver disease. The New England journal of medicine 365(2): 147-156.

3. Mallett SV (2015) Clinical Utility of Viscoelastic Tests of Coagulation (TEG/ROTEM) in Patients with Liver Disease and during Liver Transplantation. Seminars in Thrombosis and Hemostasis 41(5): 527537.

4. Abeysundara L, Mallett S, Clevenger B (2017) Point-of-Care Testing in Liver Disease and Liver Surgery. Seminars in Thrombosis and Hemostasis 43(4): 407-415.

5. Hugenholtz G, Macrae F, Adelmeijer J, Dulfer S, Porte R, et al. (2016) Procoagulant changes in fibrin clot structure in patients with cirrhosis are associated with oxidative modifications of fibrinogen. Journal of Thrombosis and Haemostasis 14(5): 1054-1066.

6. Kujovich JL (2015) Coagulopathy in liver disease: A balancing act. Hematology 2015(1): 243- 249.

7. Ghoshal K, Bhattacharyya M (2014) Overview of platelet physiology: Its hemostatic and nonhemostatic role in disease pathogenesis. The Scientific World Journal 2014(2012): 16

8. Senzolo M, Coppell J, Cholongitas E, Riddell A, Triantos C, et al. (2007) The effects of glycosaminoglycans on coagulation: a thromboelastographic study. Blood Coagul Fibrinolysis 18(3): 227236.

9. Cocero N, Bezzi M, Martini SCS (2017) Oral Surgical Treatment of Patients with Chronic Liver Disease: Assessments of Bleeding and Its Relationship with Thrombocytopenia and Blood Coagulation Parameters. J Oral Maxillofac Surg 75(1): 28-34.

10. Thakrar SV, Mallett SV (2017) Thrombocytopenia in cirrhosis: Impact of fibrinogen on bleeding risk. World J Hepatol 9(6): 318-325

11. Donohue CI, Mallett SV (2015) Reducing transfusion requirements in liver transplantation. World journal of transplantation 5(4): 165-182.

12. Mallett SV, Sugavanam A, Krzanicki DA, Patel S, Broomhead RH, et al. (2016) Alterations in coagulation following major liver resection. Anaesthesia 71(6): 657-668.

13. Yang LS, Alukaidey S, Croucher K, DD (2018) Suboptimal use of pharmacological venous thromboembolism prophylaxis in cirrhotic patients. Intern Med J.

14. Ben Ari Z, Panagou M, Patch D, Bates S, OE, et al. (1997) Hypercoagulability in patients with primary biliary cirrhosis and 
primary sclerosing cholangitis evaluated by thrombelastography. J Hepatol 26(3): 554-559.

15. Pihusch R, Rank A, Göhring P, Pihusch M, HE, et al. (2002) Platelet function rather than plasmatic coagulation explains hypercoagulable state in cholestatic liver disease. J Hepatol 37(5): 548-555.

16. Stine JG, Niccum BA, Zimmet AN, Intagliata N, Caldwell SH, et al. (2018) Increased risk of venous thromboembolism in hospitalized patients with cirrhosis due to non-alcoholic steatohepatitis. Clin Transl Gastroenterol 9(3): 140.

17. Davis JPE, Northup PG, Caldwell SH, INM (2018) Viscoelastic Testing in Liver Disease. Ann Hepatol 17(2): 205-213.

18. Massicotte L, Thibeault L, Roy A (2015) Classical Notions of Coagulation Revisited in Relation with Blood Losses, Transfusion Rate for 700 Consecutive Liver Transplantations. Semin Thromb Hemost 41(5): 538-546.

19. Tafur LA, Taura P, Blasi A, Beltran J, Martinez Palli G, et al. (2016) Rotation thromboelastometry velocity curve predicts blood loss during liver transplantation. Br J Anaesth 117(6): 741-748.

20. Kozek LSA, Ahmed AB, Afshari A, Albaladejo P, Aldecoa C, et al. (2017) Management of severe perioperative bleeding: guidelines from the European Society of Anaesthesiology: First update 2016. Eur J Anaesthesiol 34(6): 332-395.

21. De Pietri, Bianchini M, Montalti R, De Maria N, Di Maira T, et al. (2016) Thrombelastography-guided blood product use before invasive procedures in cirrhosis with severe coagulopathy: A randomized, controlled trial. Hepatology 63(2): 566-573.

\section{Your next submission with Juniper Publishers will reach you the below assets}

- Quality Editorial service

- Swift Peer Review

- Reprints availability

- E-prints Service

- Manuscript Podcast for convenient understanding

- Global attainment for your research

- Manuscript accessibility in different formats

( Pdf, E-pub, Full Text, Audio)

- Unceasing customer service

Track the below URL for one-step submission https://juniperpublishers.com/online-submission.php 